\title{
Microbial biofilm formation on silicone nasal splints: optimal time for splint removal*
}

\author{
Mustafa Acar ${ }^{1}$,Tolgahan Catli², Ilknur Dag ${ }^{3}$, Turhan San ${ }^{4}$, Cemal Cingi ${ }^{5}$ \\ 'Yunus Emre State Hospital, ENT Dept, Eskisehir, Turkey \\ ${ }^{2}$ Bozyaka Teaching and Research Hospital ENT Department, Izmir, Turkey \\ ${ }^{3}$ Electron Microscope Laboratory, Eskisehir Osmangazi University, Eskisehir, Turkey \\ ${ }^{4}$ Istanbul Medeniyet University, ENT Department, Istanbul, Turkey \\ ${ }^{5}$ Eskisehir Osmangazi University, Medical Faculty, Department of Otorhinolaryngology, Eskisehir, Turkey
}

Rhinology 52: 371-375, 2014

DOI:10.4193/Rhino13.156

*Received for publication:

Oktober 3, 2013

Accepted: February 16, 2014

\begin{abstract}
Objectives: Biofilms are sessile communities associated with persistent infections and are resistant to conventional therapeutic strategies. They survive on the surface of various inorganic medical devices and cause serious medical problems.

Methods: We recruited 25 patients who underwent nasal surgery between January and May 2013. All patients received silicone splints at the conclusion of the procedure. Pieces of the splints were collected 48,72 and $96 \mathrm{~h}$ post-surgery and prepared for scanning electron microscopy evaluation to assess biofilm formation.
\end{abstract}

Results: Biofilm was observed in 3, 14 and 25 of the 25 samples at 48,72 and $96 \mathrm{~h}$, respectively. The differences in the proportions of the samples with biofilm formation at each time point $(48,72$, and $96 \mathrm{~h})$ were statistically significant.

Conclusion: Our data demonstrated that biofilm formation on silicone splints increases significantly after $48 \mathrm{~h}$ following placement. Although packing may reduce complications, surgeons must consider the potential hazards of packing materials, such as biofilm formation at $48 \mathrm{~h}$ post-surgery.

Key words: biofilm, scanning electron microscopy, nasal splints, antibiotics, complications

\section{Introduction}

Nasal septal surgery is a common otorhinolaryngological procedure and may be performed separately or as part of a surgical plan ${ }^{(1)}$. As with most surgical procedures, septoplasty has potential complications which include the formation of synechiae, bleeding and septal haematoma ${ }^{(2)}$. Various intraoperative and postoperative measures have been developed to prevent these complications, including proper subpericondrial elevation, transseptal fixation sutures and postoperative nasal packing. Of these measures, nasal packing is the most controversial. Several complications have been reported following nasal packing including dysphagia, aspiration, airway obstruction, hypoventilation, hypoxemia, local and/or general infections, the feeling of discomfort and reduced quality of life ${ }^{(3,4)}$.

Antibiotic ointment applied to the packing material and prophylactic enteral or parenteral antibiotics have been used to prevent nasal packing-related infections. However, local or general infection-related complications, such as vestibulitis, cellulitis, septal abscess, sinusitis, meningitis, cavernous sinus thrombosis and intracranial abscess may occur despite the use of preventive strategies ${ }^{(5)}$. Most surgeons recommend not using packing if possible; however, when the procedure is necessary, there are no objective data to provide guidelines regarding the optimal removal time. Several surgeons strongly recommend removing the nasal packs in a timely fashion to reduce infectionrelated problems following nasal septal surgery. A recent report 
suggests that silicone sheets that sit on both sides of the septum to hold it straight and that are left in place for up to $96 \mathrm{~h}$ are useful for preventing postoperative complications ${ }^{(6)}$.

Since the relationship between microbial biofilms and the development of various human infections was revealed (7), clinicians have been mindful of the role played by biofilms in the aetiology of microbiological manifestations. Biofilm communities are unique and highly resistant to standard antibiotic therapies. They have evolved several mechanisms of antibiotic resistance distinct from those of planktonic bacteria. We measured biofilm formation on silicone nasal splints used at the conclusion of nasal septal surgery to determine the optimal timeframe for the removal of nasal packing material.

\section{Materials and methods}

Our prospective, nonrandomised controlled study included 25 consecutive patients with nasal septal deviation and a history of nasal obstruction that were scheduled to undergo nasal septoplasty at Eskisehir Yunus Emre State Hospital ENT Department. The ethics committee of Eskisehir Osmangazi University approved the study protocol, and all participants provided informed consent. Standard physical examinations with anterior rhinoscopy and rigid nasal endoscopy were performed, and patients who had concurrent sinusitis, allergic rhinitis, or nasal polyposis were excluded from the study.

\section{Surgery}

Surgical procedures were performed under local anaesthesia by the same surgeon. All participants were premedicated using $10 \mathrm{mg}$ intramuscular diltiazem hydrochloride and received $1 \mathrm{~g}$ intravenous co-amoxiclav for antimicrobial prophylaxis. Gauzes soaked in xylometazoline $0.1 \%$ were used for nasal mucosal decongestion. We injected 2-mL lidocaine $\mathrm{HCl} 1 \%$ with adrenaline 1:100,000 to achieve vascular haemostasis. Briefly, our approach involved a Killian incision, creation of subpericondrial tunnels, posterior chondrotomy, osteotomy if necessary and septal reconstruction. Nasal packing using silicone splints that contained no lubricant agent or topical antimicrobial was performed at the conclusion of surgery. One silicone splint was applied to each nasal cavity and left in place for $96 \mathrm{~h}$. In general, nasal splint removal times vary widely, usually depending on the surgeon and the patients' status. We removed the splints at 96 $\mathrm{h}$ to allow us to compare biofilm formation at three time points (48, 72 and $96 \mathrm{~h})$.

\section{Sample collection}

Patients were hospitalised for $24 \mathrm{~h}$ and were administered co-amoxiclav ( $2 \mathrm{~g}$ per day perorally). The first samples were collected under sterile conditions from the most caudal end of the splints $48 \mathrm{~h}$ after placement. The second and third samples were under taken under sterile conditions at 72 and 96 h, respectively. All samples were immediately prepared for scanning electron microscopy evaluation.

\section{Scanning electron microscopy}

The silicone samples were immediately placed in $2.5 \%$ glutaraldehyde (prepared in $0.1 \mathrm{M}$ phosphate buffer, $\mathrm{pH} 7.4$ for $24 \mathrm{~h}$ at $4^{\circ} \mathrm{C}$ as a prefixation step). The samples were rinsed twice with $0.1 \mathrm{M}$ phosphate buffer ( $\mathrm{pH} 7.4$ ) and postfixed in 1\% osmium tetroxide for $1 \mathrm{~h}$ at room temperature and then rinsed again with distilled water. Following that, the specimens were dehydrated in graduated concentrations of ethyl alcohol (30, 50, 70, 90 and 96\%) for $15 \mathrm{~min}$ each followed by absolute alcohol for $30 \mathrm{~min}$. After that, the specimens were dried using a Critical Point Dryer (Polaron CPD, Quorum Technologies, East Sussex, UK). Carbon conductive paint and gold coat (Polaron SC762-Sputter Coater, Quorum Technologies) were used for mounting and specimen coating, respectively. Finally, each specimen was examined using a JEOL scanning electron microscope (JEOL JSM05600LV, Jeol Ltd., Tokyo, Japan). The surface of each sample was scanned systematically. We defined biofilm architecture as dense accumulations of microorganisms within an amorphous matrix, according to Chole and Faddis ${ }^{(8)}$.

\section{Statistical analyses}

We used t-tests to detect significant differences in the ratio of biofilm formation between time periods.

\section{Results}

Of the 25 patients, 16 were male and 9 were female, and the age range of participants was 24-60 years (mean age, 36.3 years).

The SEM findings revealed that 3 (12\%), 14 (56\%) and 25 (100\%) of the 25 silicone samples exhibited microbial biofilm formation on their surface at 48, 72 and $96 \mathrm{~h}$, respectively (Figures 1,2, 3 and 4 ; Table 1). The percentage of biofilm formation was significantly different between the 48- and 72-h, between 48 and $96 \mathrm{~h}$ and between the 72- and 96-h time points ( $p<0.001$ for both). No intra- or postoperative complications occurred.

\section{Discussion}

Nasal septal procedures may conclude with nasal packing, and various types of materials have been used for this task. Although these endonasal materials play an important role during the postoperative period by preventing bleeding and septal haematoma formation, they are synthetic and "foreign" objects in the body. As with all foreign materials, nasal packs may cause infection. Otherwise, nasal airway procedures are considered to be contaminated and may contribute to postoperative infectious complications ${ }^{(9)}$. The risk of infection is the primary reason for use of postoperative prophylactic antibiotics. 


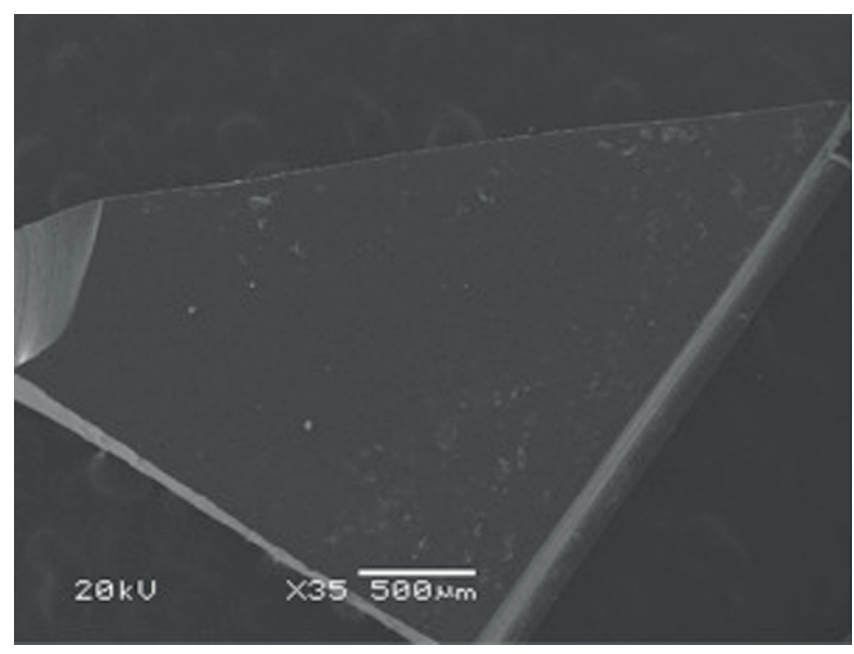

Figure 1. Scanning electron micrograph of a slicone splint at low (X35) magnification (48 h).

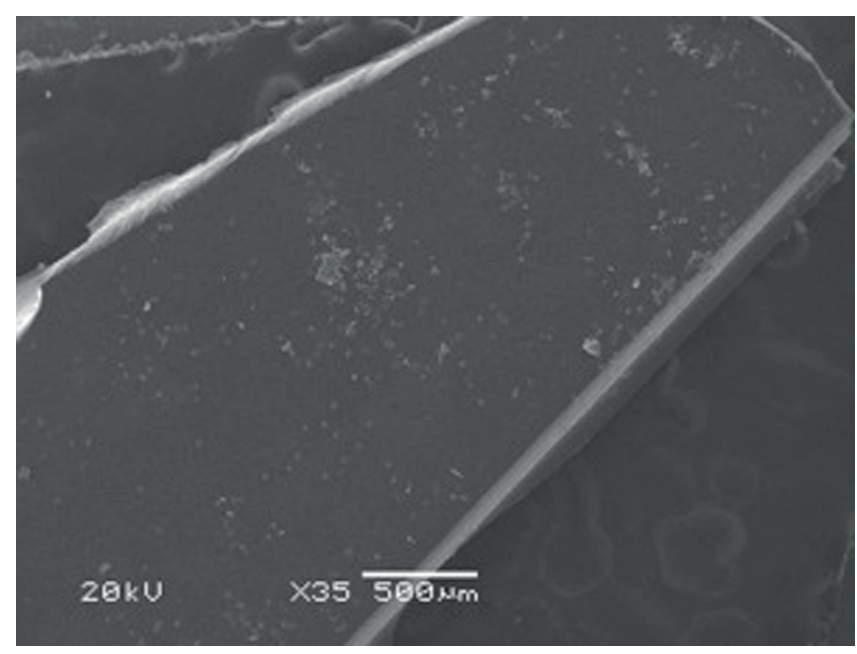

Figure 2. Scanning electron micrograph of a slicone splint with biofilm at low (X35) magnification (72 h).

According to Rechtweg et al. ${ }^{(10)}$, the most common reasons for prophylactic antibiotic use following rhinological procedures are to prevent postoperative infection (60.4\%), avoid toxic shock syndrome (31.5\%) and to avert medicolegal issues (4.9\%). We used prophylactic antibiotics in our study to prevent infection. Furthermore, guidelines for the optimal time to remove nasal packing are critical because bacteraemia increases within 48 h of placement ${ }^{(5)}$, and patients may experience significant discomfort when packing is left in place for 2 or more days ${ }^{(1)}$. We left the silicone splints in place for $96 \mathrm{~h}$, which is a common timeframe in general practice; however, no randomised clinical trials have investigated the optimal time for the removal of nasal packing. Surgeons are divided on the efficacy of nasal packing; some argue that it is not necessary and septal suturing achieves the same goal ${ }^{(11)}$, others continue to use nasal packing to

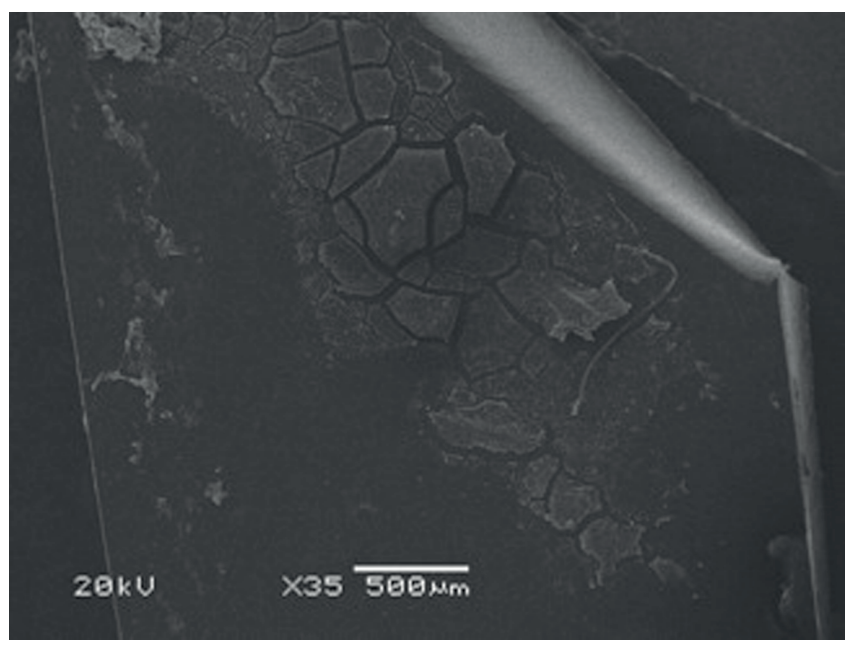

Figure 3. Scanning electron micrograph of a slicone splint with biofilm at low (X35) magnification (96 h).

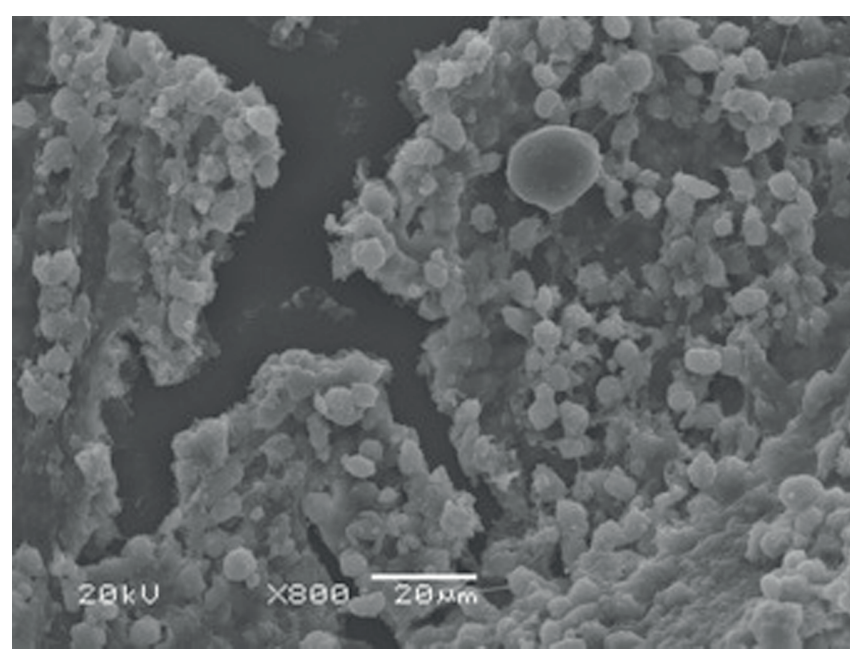

Figure 4. Scanning electron micrograph of a slicone splint with biofilm at high (X800) magnification (96 h).

prevent potential complications. Nonetheless, nasal packs left in place for more than $48 \mathrm{~h}$ may be uncomfortable for the patient and increase the risk of infection. As a personal preference of the senior author of the present study, we used a silicone splint as the packing material. Although postoperative synechiae formation did not occur in our patient group, this finding may not be considered an advantage of silicone splints because most randomised clinical trials have shown that silicone splints cause a significant increase in postoperative pain with no sufficient evidence of a decrease in the rates of intranasal adhesions or other clinically significant complications ${ }^{(12,13)}$.

Our findings in silicone nasal splints are consistent with those of previous studies showing that the rate of biofilm formation in nasal packing increases after $48 \mathrm{~h}$. Biofilm cells are physiologically distinct from those existing planktonically on several 
Table 1. Patients and SEM findings.

\begin{tabular}{|c|c|c|c|c|}
\hline \multirow{2}{*}{$\begin{array}{c}\text { Patient No \& } \\
\text { Gender }\end{array}$} & \multirow{2}{*}{ Age } & \multicolumn{3}{|c|}{ Time Period (Hour) } \\
\hline & & 48 & 72 & 96 \\
\hline $1: F$ & 39 & - & - & + \\
\hline $2: F$ & 29 & - & - & + \\
\hline $3: M$ & 33 & - & - & + \\
\hline $4: M$ & 37 & - & + & + \\
\hline $5: F$ & 45 & - & - & + \\
\hline $6: F$ & 35 & - & + & + \\
\hline $7: M$ & 27 & - & + & + \\
\hline $8: M$ & 46 & - & + & + \\
\hline $9: M$ & 24 & + & + & + \\
\hline $10: F$ & 28 & - & + & + \\
\hline $11: M$ & 28 & - & - & + \\
\hline $12: M$ & 29 & - & - & + \\
\hline $13: M$ & 58 & - & - & + \\
\hline $14: F$ & 37 & - & + & + \\
\hline $15: F$ & 54 & - & + & + \\
\hline $16: M$ & 60 & - & - & + \\
\hline $17: M$ & 41 & + & + & + \\
\hline $18: M$ & 35 & & + & + \\
\hline $19: M$ & 49 & & + & + \\
\hline $20: F$ & 36 & & - & + \\
\hline $21: M$ & 24 & + & + & + \\
\hline $22: M$ & 28 & & + & + \\
\hline $23: M$ & 26 & & - & + \\
\hline $24: F$ & 33 & & - & + \\
\hline $25: M$ & 27 & & + & + \\
\hline
\end{tabular}

* M: Male, F: Female

** (-) : No biofilm formation, (+) : Biofilm formation levels, such as gene transcription, phenotype and resistance to antibacterial agents ${ }^{(14,15)}$. The high level of antibiotic resistance exhibited by biofilm occurs via several mechanisms, including (i) decreased penetration or diffusion of antimicrobial agents into biofilms, (ii) increased activity of multidrug efflux pumps, (iii) involvement of quorum-sensing systems, (iv) starvation or stress responses and ( $v$ ) genetic switches that transform susceptible planktonic cells into antibiotic-resistant persisters ${ }^{(16)}$. Strategies to eradicate biofilm are limited. The most effective method for removing biofilm is excision of the affected tissue or foreign material at the optimal time ${ }^{(8)}$. Our findings show that despite antibiotic prophylaxis, infection-related complications may occur at $48 \mathrm{~h}$ following the placement of nasal silicone splints, and infection is likely to occur at $96 \mathrm{~h}$ following packing. Our findings are consistent with previous reports that $48 \mathrm{~h}$ is the optimal time for packing removal to prevent infection-related complications and minimise patient discomfort. The fact that none of our patients experienced postoperative complications limits our ability to draw conclusions regarding the relationship between biofilm formation and the risk of postoperative complications. Thus, more studies of the association between biofilm and postoperative complications are needed.

In conclusion, our data showed that biofilm formation on the surface of nasal silicone splints increases significantly after 48

h. Although postoperative interventions, such as nasal packing with silicone splints, may reduce the frequency of surgical complications, surgeons must be aware of the potential hazards, such as biofilm formation $48 \mathrm{~h}$ after application.

\section{Acknowlegdement}

This work has been approved by the ethical committee of Eskisehir Osmangazi University (Project no. 201341D04). With the exception of data collection, the preparation of this paper, including design and planning, was supported by the Continuous Education and Scientific Research Association.

\section{Authorship contribution}

MA: Surgical procedure; TÇ: Manuscript writing; ID: SEM study; TS: Data collection, analysis; CC: Data control, Revised manuscript

Dr. M. Acar takes responsibility for the integrity of the content of the paper.

\section{Conflicts of Interest}

Authors declare that there is no competing interest.

\section{References}

1. Hajioannou JK, Bizaki A, Fragiadakis G, Bourolias C, Spanakis I, Chlouverakis G, Bizakis J. Optimal time for nasal packing removal after septoplasty. A comparative study. Rhinology. 2007; 45: 68-71.

2. Ibrahimov M, Yilmaz M, Akil F, Tarhan O, Kaya N, Ozturk O. Nasal septal cyst: a rare complication of nasal septal surgery. J Craniofac Surg. 2012; 23: 1933-1934.
3. Watson MG, Campbell JB, Shenoi PM. Nasal surgery: does the type of nasal pack influence the results? Rhinology. 1989; 27: 105111

4. Muluk NB, Oğuztürk O, Ekici $A$, Koç 
C. Emotional effects of nasal packing measured by the Hospital Anxiety and Depression Scale in patients following nasal surgery. J Otolaryngol. 2005; 34: 172-177.

5. Caniello M, Passerotti GH, Goto EY, Voegels $\mathrm{RL}$, Butugan O. Antibiotics in septoplasty: is it necessary?. Braz J Otorhinolaryngol. 2005; 71: 734-738.

6. Campbell JB, Watson MG, Shenoi PM. The role of intranasal splints in the prevention of post-operative nasal adhesions. J Laryngol Otol. 1987; 101: 1140-1143.

7. Wilson M. Bacterial biofilms and human disease. Sci Prog. 2001; 84: 235-254.

8. Chole RA, Faddis BT. Evidence for microbial biofilms in cholesteatomas. Arch Otolaryngol Head Neck Surg. 2002; 128: 1129-1133.

9. Dellinger EP. Surgical Infections and choice of antibiotics. In: Townsend CM, Beauchamp RD, Evers BM, Mattox KL. Sabiston textbook of surgery. 16th ed. New
York; 264-279.

10. Rechtweg JS, Paolini RV, Belmont BJ, Wax MK. Postoperative antibiotic use of septoplasty: A survey of practice habits of the membership of the American Rhinologic Society. Am J Rhinol 2001; 22: 315-320.

11. Ozkırış M, Kapusuz Z, Saydam L. Comparison of nasal packs with transseptal suturing after nasal septal surgery.. Am J Otolaryngol. 2013; 34: 308-311.

12. Ardehali MM, Bastaninejad S. Use of nasal packs and intranasal septal splints following septoplasty. Int J Oral Maxillofac Surg 2009; 38: 1022-1024.

13. Tang S, Kacker A. Should intranasal splints be used after nasal septal surgery? Laryngoscope. 2012; 122: 1647-1678.

14. Sanderson AR, Leid JG, Hunsaker D. Bacterial biofilms on the sinus mucosa of human subjects with chronic rhinosinusitis. Laryngoscope. 2006; 116: 1121-1126.

15. Jeffrey D. Suh, Noam A. Cohen and James
N. Palmer. Biofilm in chronic rhinosinusitis. Curr Opin Otolaryngol Head Neck Surg. 2010; 18: 27-31.

16. Leid JG. Bacterial biofilms resist key host defenses. Microbe. 2009; 4: 66-70.

\section{Tolgahan Catli}

Bozyaka Teaching and Research Hospi-

tal ENT Department

Izmir

Turkey

Tel: $+90-232-2505050$

Fax: +90-232-250 5051

E-mail: tcatli80@hotmail.com

\section{ADVERTISEMENT}

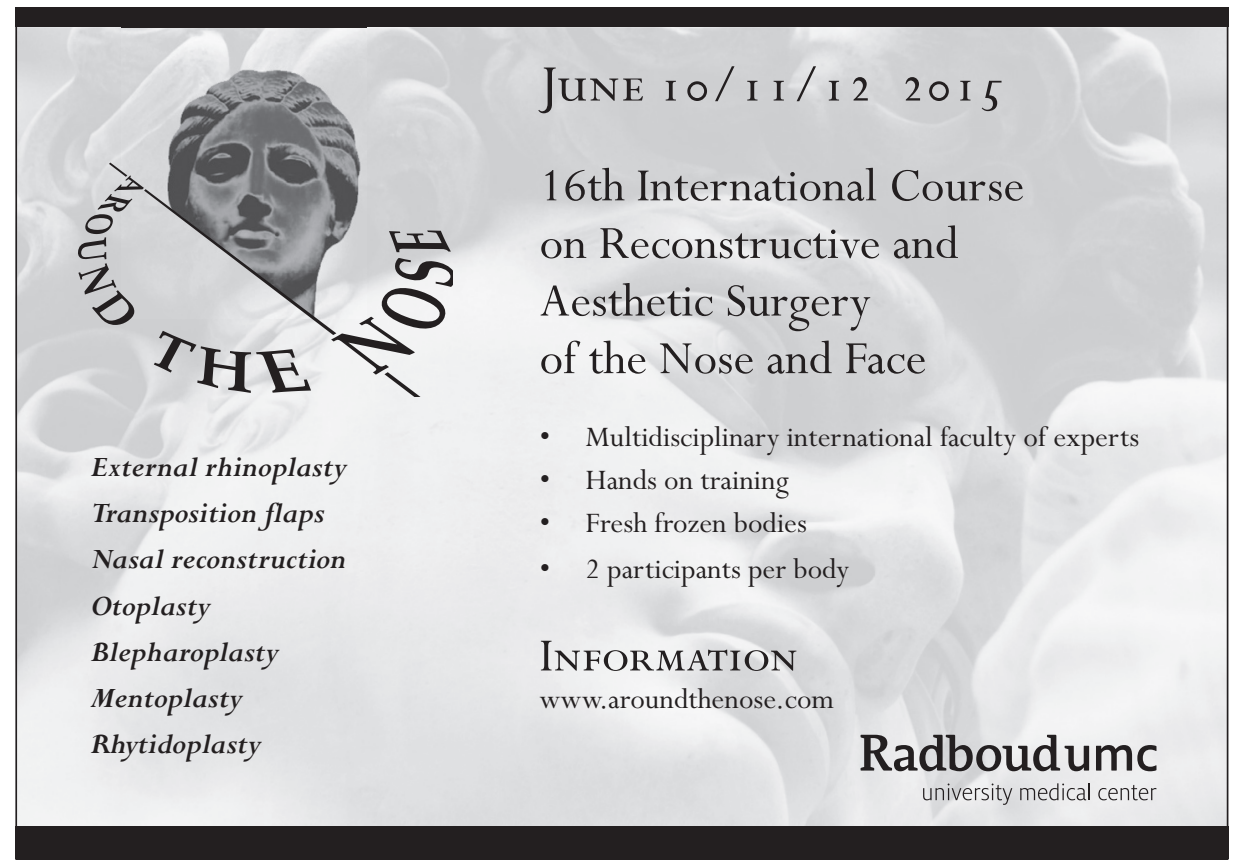

